\title{
Three-fold enhancement of in-plane thermal conductivity of borophene through metallic atoms intercalation
}

\author{
Yanxiao $\mathrm{Hu}^{1 \mathrm{a}}$, Yan Yin ${ }^{1 \mathrm{a}}$, Shichang $\mathrm{Li}^{1}$, Hangbo Zhou ${ }^{2}$, Dengfeng $\mathrm{Li}^{1 *}$, Gang \\ Zhang $^{2 *}$
}

1. School of Science, Chongqing University of Posts and Telecommunications, Chongqing, 400065, China.

2. Institute of High Performance Computing, A*STAR, 138632, Singapore

${ }^{a}$ Y. H. and Y. Y. contributed equally to this work.

*Corresponding authors. E-mail: lidf@,cqupt.edu.cn(D.F.Li); zhangg@ihpc.a-star.edu.sg (G. Zhang) 


\section{S1. Polarization effect on thermal transport properties}

As shown in Fig.S3, there exist two types of boron atoms in both $\mathrm{AlB}_{6}$ and $\delta_{4}$ sheet. In order to numerically analyze the charge transfer, we calculate the Born effective charges matrix $\left(\mathrm{Z}^{*}\right)$ and the dielectric constants matrix $(\epsilon)$ of $\mathrm{AlB}_{6}$ and $\delta_{4}$ sheet, as shown in Table $\mathrm{S} 1$.

At the limit of zero wavevector $(q \rightarrow 0)$, the dynamical matrix $D_{a \beta}^{i j}$ can be separated into the contributions of short-range covalent bonding and the long-range dipole-dipole interaction as following:

$$
D_{a \beta}^{i j}(q \rightarrow 0)=D_{\text {short }}^{i j \beta}(q=0)+D_{\text {long }_{\alpha \beta}}^{i j}(q \rightarrow 0)
$$

Here, the $D_{\text {short } \alpha \beta}^{i j}$ term can be obtained after the Fourier transform of the second interatomic force constants matrix and $D_{\text {long }}^{i j \beta}$ term given by:

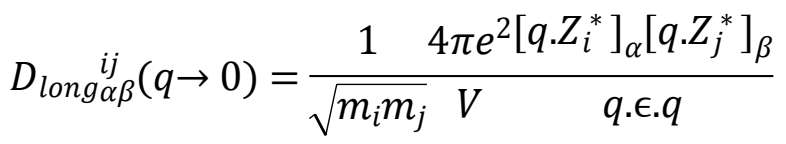

Here $m, e$ and $V$ are atomic mass, electron charge and volume of the unit cell, respectively. $i$ and $\alpha$ denote the atom index and vector direction. When the wavevector $q$ is away from $\Gamma$ point, the contribution of $D_{\text {long }}^{i j \beta}$ term to dynamical matrix $D_{a \beta}^{i j}$ quickly vanishes.

The obvious polarization effect in $\delta_{4}$ sheet on thermal conductivity can be understood through the relaxation time (see Fig.S4(a)-(b)). Considering the polarization 
effect, there is a pronounced increase in relaxation time of $\delta_{4}$ sheet in low frequency region (near $1.3 \mathrm{THz}$ ). 

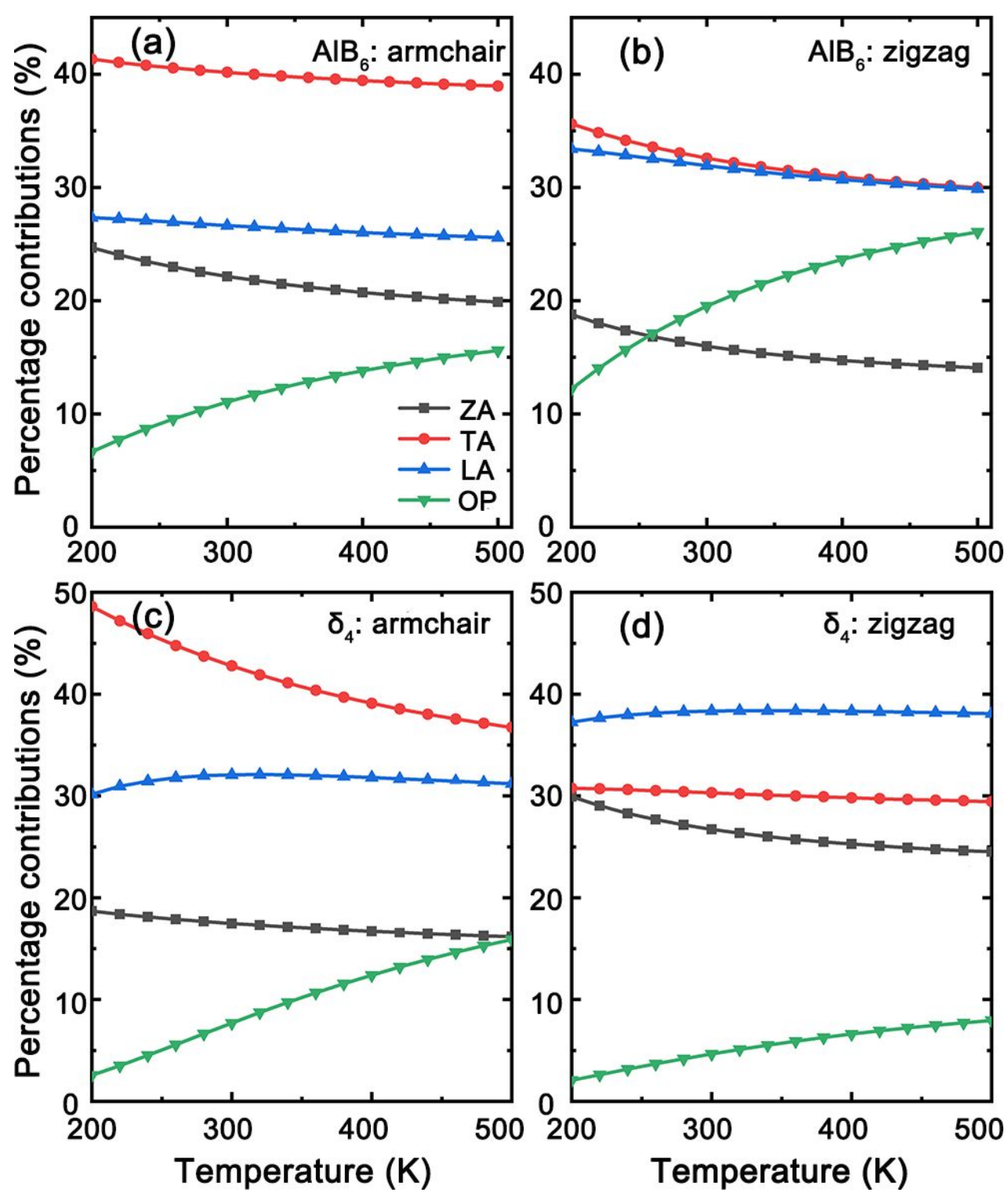

Figure S1. The temperature dependent contribution of acoustic and optical branches of $\mathrm{AlB}_{6} / \delta_{4}$ along (a)/(c) armchair and (b)/(d) zigzag direction, respectively. 


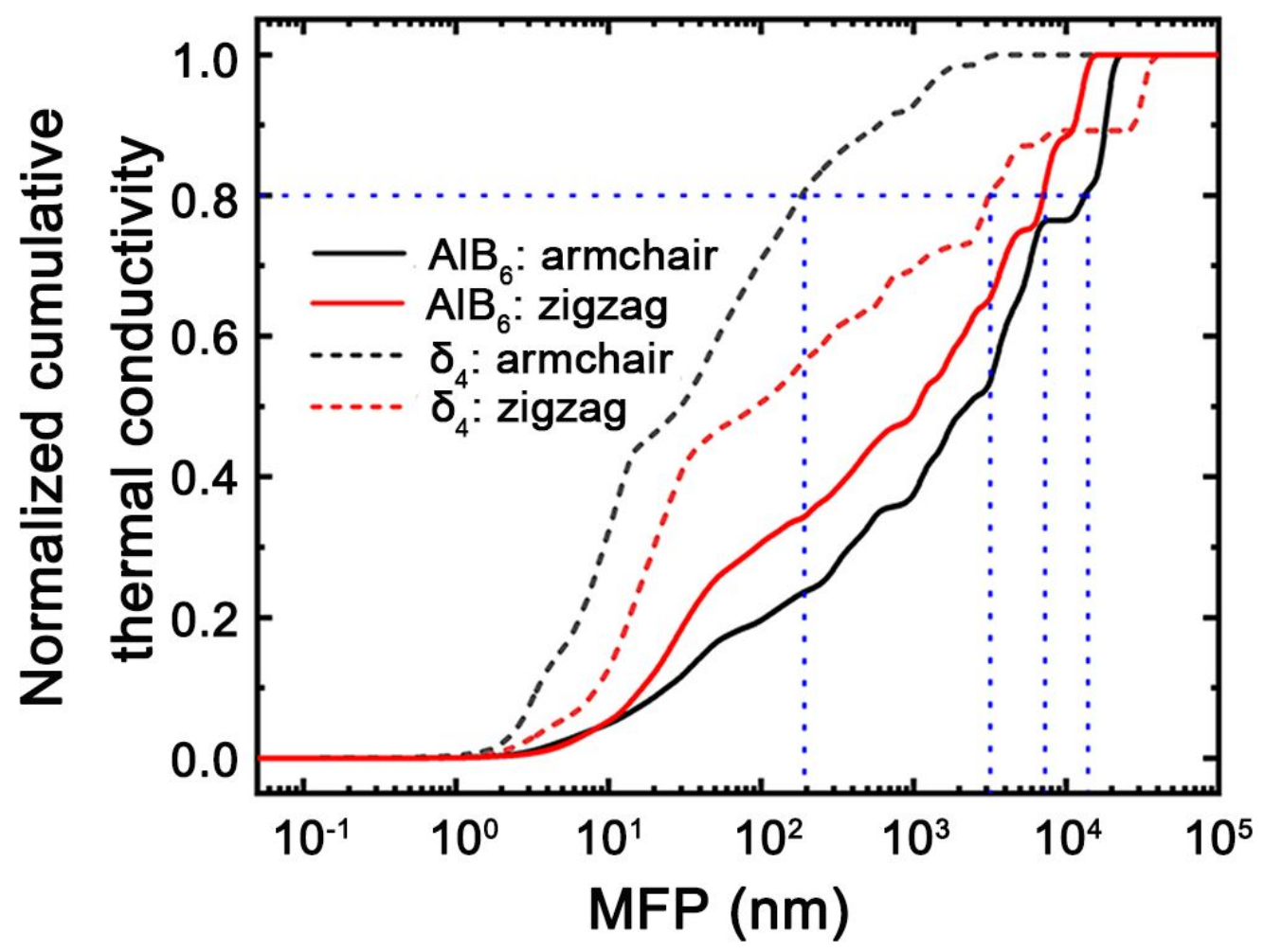

Figure S2. The cumulative thermal conductivity of $\mathrm{AlB}_{6}$ and $\delta_{4}$ as a function of mean free path of phonons. 

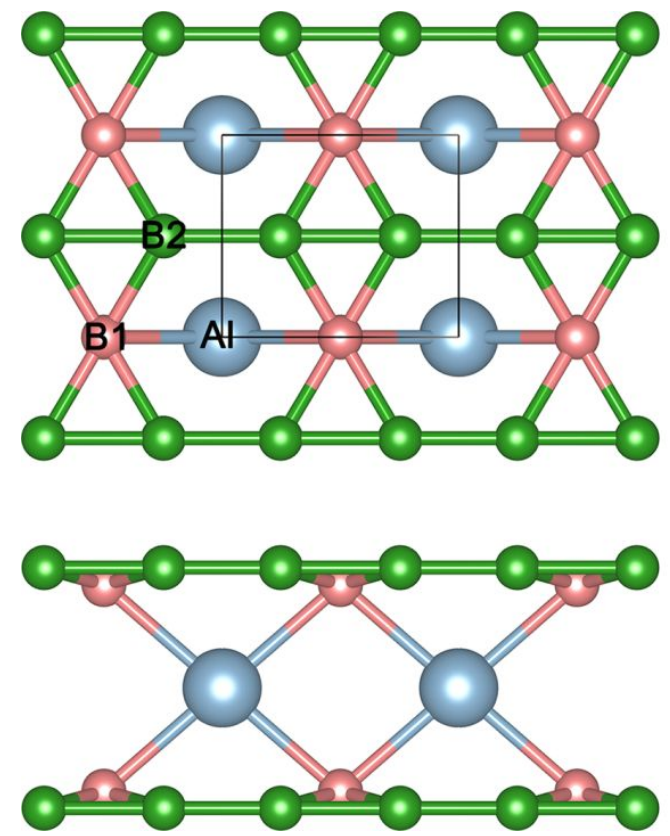
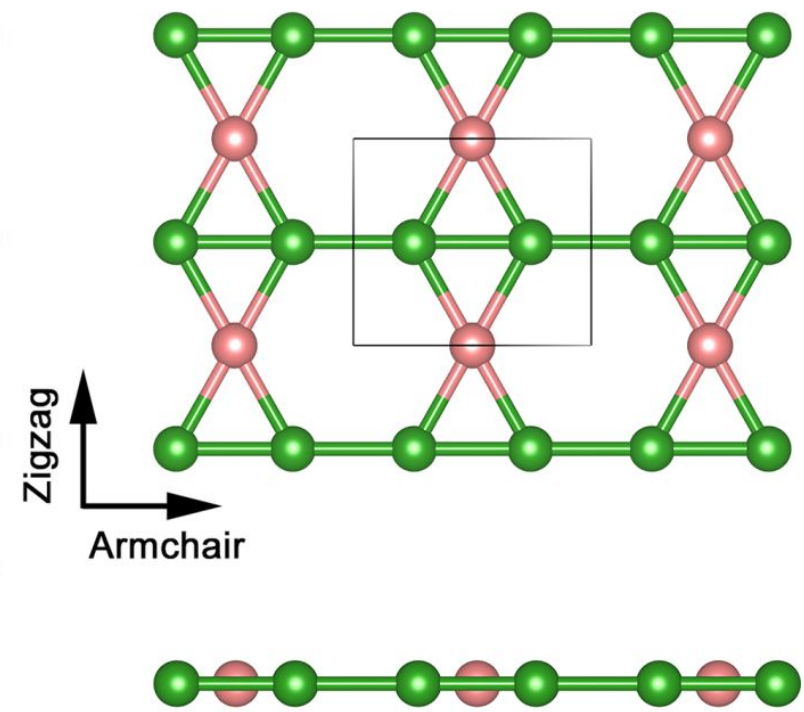

Figure S3. Crystal structures of $\mathrm{AlB}_{6}$ (left) and $\delta_{4}$ sheets (right). 


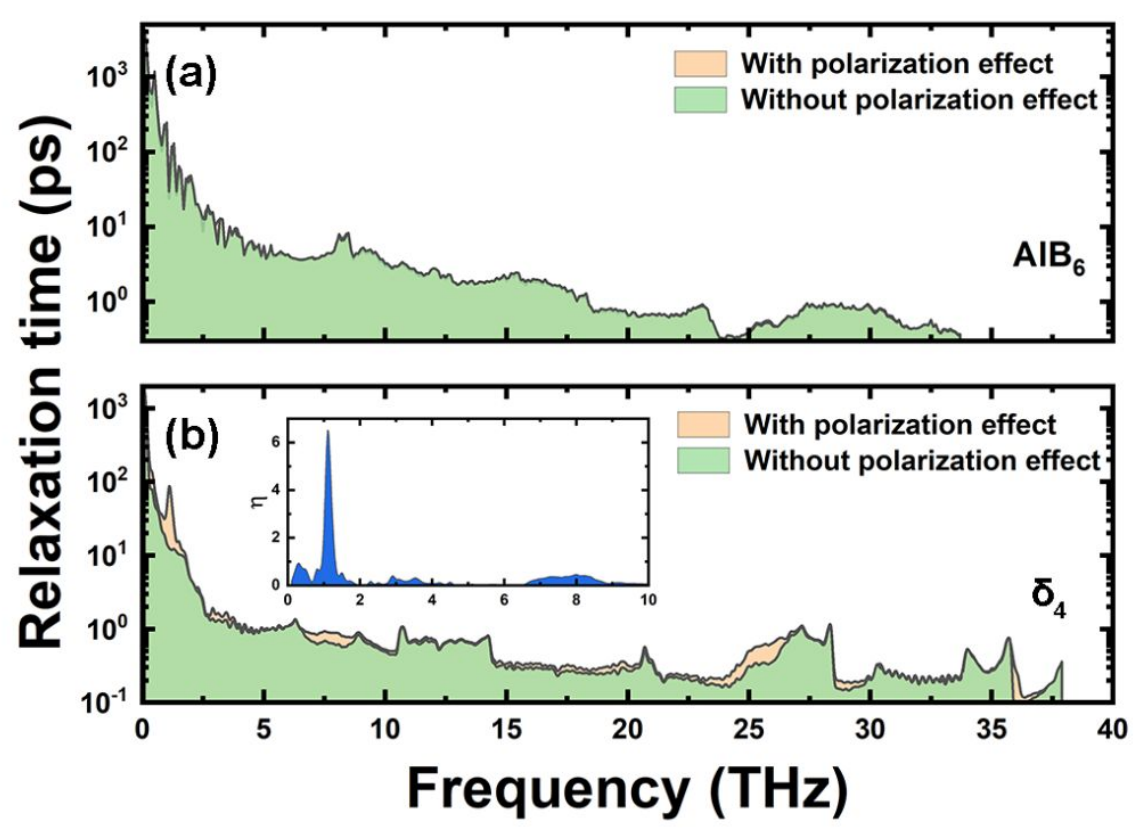

Figure S4. The polarization effect on phonon relaxation time of (a) $\mathrm{AlB}_{6}$ and (b) $\delta_{4}$ sheets at $300 \mathrm{~K}$. The factor $\eta$ in insert is defined as $\frac{\tau_{\text {with }}-\tau_{\text {without }}}{\tau_{\text {without }}} . \tau_{\text {with }}$ and $\tau_{\text {without }}$ are the relaxation time with and without long-range dipole-dipole interaction, respectively. 
Table S1. The phonon group velocity of $\mathrm{AlB}_{6}$ and $\delta_{4}$ at $\Gamma$ point.

\begin{tabular}{ccccc}
\hline \hline & \multicolumn{2}{c}{$v_{T A}(\mathrm{Km} / \mathrm{s})$} & \multicolumn{2}{c}{$v_{L A}(\mathrm{Km} / \mathrm{s})$} \\
\cline { 2 - 5 } & Armchair & Zigzag & Armchair & Zigzag \\
\hline$\delta_{4}$ & 6.50 & 4.05 & 9.77 & 18.97 \\
$\mathrm{AlB}_{6}$ & 10.07 & 10.00 & 16.75 & 15.51 \\
\hline
\end{tabular}


Table S2. The contribution of different acoustic branches and optical branches to thermal conductivity of $\mathrm{AlB}_{6}$ and $\delta_{4}$ at $300 \mathrm{~K}$.

\begin{tabular}{lccc}
\hline \hline Directions & Phonon modes & \multicolumn{2}{c}{ Contribution (\%) } \\
\cline { 2 - 3 } & & AlB 6 & $\delta_{4}$ \\
\hline ZA & 22.2 & 17.5 \\
TA & & 42.8 \\
& LA & 40.2 & 32.1 \\
& OP & 26.6 & 7.6 \\
ZA & 11.0 & 26.7 \\
Zigzag & TA & 16.0 & 30.3 \\
& LA & 32.6 & 38.3 \\
\hline \hline
\end{tabular}


Table S3. Born effective charges $\left(Z^{*}\right)$ and the dielectric constants of $\mathrm{AlB}_{6}$ and $\delta_{4}$ sheet.

\begin{tabular}{|c|c|c|c|c|c|}
\hline Compounds & Components & $\mathrm{Z}^{*}\left(\mathrm{~B}_{1}\right)$ & $\mathrm{Z}^{*}\left(\mathrm{~B}_{2}\right)$ & $\mathrm{Z}^{*}(\mathrm{Al})$ & $\epsilon$ \\
\hline \multirow[t]{3}{*}{$\mathrm{AlB}_{6}$} & $x x$ & -1.924 & 0.398 & 2.263 & 6.741 \\
\hline & yy & -0.966 & 0.173 & 1.242 & 8.735 \\
\hline & $\mathrm{zz}$ & -0.010 & -0.025 & 0.119 & 1.419 \\
\hline \multirow[t]{3}{*}{$\delta_{4}$ sheet } & $\mathrm{xx}$ & -1.187 & 0.596 & -- & 2.203 \\
\hline & yy & 1.488 & -0.744 & -- & 2.385 \\
\hline & $\mathrm{zZ}$ & 0.014 & 0.007 & -- & 1.176 \\
\hline
\end{tabular}

